\title{
Control of Exciton Valley Coherence in Transition Metal Dichalcogenide Monolayers
}

\author{
G. Wang, ${ }^{1}$ X. Marie, ${ }^{1}$ B. L. Liu, ${ }^{2, *}$ T. Amand, ${ }^{1}$ C. Robert, ${ }^{1}$ F. Cadiz, ${ }^{1}$ P. Renucci, ${ }^{1}$ and B. Urbaszek ${ }^{1, \dagger}$ \\ ${ }^{1}$ Université de Toulouse, INSA-CNRS-UPS, LPCNO, 135 Avenue Rangueil, 31077 Toulouse, France \\ ${ }^{2}$ Beijing National Laboratory for Condensed Matter Physics, Institute of Physics, Chinese Academy of Sciences, \\ P.O. Box 603, Beijing 100190, Peoples Republic of China
}

(Received 2 June 2016; published 25 October 2016)

\begin{abstract}
The direct gap interband transitions in transition metal dichalcogenide monolayers are governed by chiral optical selection rules. Determined by laser helicity, optical transitions in either the $K^{+}$or $K^{-}$valley in momentum space are induced. Linearly polarized laser excitation prepares a coherent superposition of valley states. Here, we demonstrate the control of the exciton valley coherence in monolayer $\mathrm{WSe}_{2}$ by tuning the applied magnetic field perpendicular to the monolayer plane. We show rotation of this coherent superposition of valley states by angles as large as $30^{\circ}$ in applied fields up to $9 \mathrm{~T}$. This exciton valley coherence control on the ps time scale could be an important step towards complete control of qubits based on the valley degree of freedom.
\end{abstract}

DOI: 10.1103/PhysRevLett.117.187401

Atomically thin layers of van der Waals bonded materials open up new possibilities for fundamental physics in 2D systems and for new applications [1-3]. Here, the group-VI transition metal dichalcogenides (TMDCs) of the form $M X_{2}$, where $M=\mathrm{Mo}, \mathrm{W}$ and $X=\mathrm{S}$, Se stand out: These indirect semiconductors in bulk form become direct semiconductors when thinned down to one monolayer (ML) [4-12]. The valence and conduction band extrema of a ML reside at the $K$ point of the Brillouin zone. Current research interest is stimulated by their strong light-matter interaction and the possibility of using the valley index as an information carrier and for exciting fundamental physics experiments [13-18]. Because of the reduction of dielectric screening and the large effective carrier masses in TMDC monolayers, the light-matter interaction is dominated by the excitons (Coulomb bound electron-hole pairs), with binding energies up to several hundred meV [19-25]. Using $\sigma^{+}\left(\sigma^{-}\right)$polarized excitation, the optical excitation of carriers in the $K^{+}\left(K^{-}\right)$valley results in formation of an exciton with pseudospin $|+1\rangle(|-1\rangle)$ [26-28].

A basic requirement for quantum information processing experiments using the valley degree of freedom is the ability to completely control the state of a single qubit, as demonstrated for electron spins in quantum dots [29]. A universal single qubit gate is realized by a rotation of a single spin, for example, by any angle about an arbitrary axis. One strategy for qubit manipulation is to use picosecond or femtosecond laser pulses, permitting an arbitrary rotation to be completed within one spin precession period. Concerning the valley degree of freedom, a first important

Published by the American Physical Society under the terms of the Creative Commons Attribution 3.0 License. Further distribution of this work must maintain attribution to the author(s) and the published article's title, journal citation, and DOI. step was the recent demonstration in $\mathrm{ML} \mathrm{WSe}_{2}$ of the generation of a superposition of two valley states as $|X\rangle=1 / \sqrt{2}(|+1\rangle+|-1\rangle)$. This is optically achieved by linearly polarized excitation $\sigma_{X}$, which results in a strongly linearly polarized neutral exciton $\left(X^{0}\right)$ emission [30-32]. In ML TMDCs, the optical excitation can be at much higher energy than the transition energy [32]; i.e., strictly resonant excitation or detection of the coherent exciton states is not required. This allows us to easily filter scattered laser light and detect only exciton emission, in contrast, for example, to resonant exciton manipulation required in GaAs quantum wells [33-35]. Despite these favorable conditions for coherent manipulation of valley states, so far, a demonstration of tunable control of exciton valley coherence is lacking.

In this Letter, we demonstrate that the neutral exciton valley coherence in monolayer $\mathrm{WSe}_{2}$ can be controlled by an external magnetic field applied vertically to the sample plane. We optically initialize a superposition of valley states, rotate this superposition by a finite angle that we tune with a magnetic field, and subsequently, detect the new superposition state through its polarization in photoluminescence (PL) emission. In the absence of external fields, the electronic states related by time reversal in the $K^{+}$and $K^{-}$valleys are degenerate. The valley exciton degeneracy can be lifted by a longitudinal magnetic field [36-41] or the optical Stark effect $[42,43]$. In our experiment, the external magnetic field lifts the valley degeneracy and results in a change of the oscillation frequency of the coherent superposition of valley states. This corresponds to a rotation of valley coherence (i.e., the exciton pseudospin) and we clearly measure this rotation in our experiments with angles up to $30^{\circ}$ at $B=9 \mathrm{~T}$. This type of quantum beat process was observed initially in atoms and molecular systems [44] and, then, intensely investigated for excitons in low dimensional GaAs structures by time-resolved optical techniques 
[33-35]. Whereas, in other material systems, coherent manipulation is necessarily a two pulse experiment to read and write the quantum state, here, we show that, in ML TMDCs, these experiments can be carried out with simple $\mathrm{cW}$ excitation and PL detection.

Samples and experimental setup.-The $\mathrm{WSe}_{2}$ ML flakes are prepared by micromechanical cleavage of a bulk crystal (from 2D semiconductors) on $\mathrm{SiO}_{2} / \mathrm{Si}$ substrates. The experiments are carried at $T=4 \mathrm{~K}$ and, in magnetic fields, up to $\pm 9 \mathrm{~T}$ in Faraday configuration in a confocal microscope as shown in Fig. 1(a). The excitation or detection spot diameter is $\approx 1 \mu \mathrm{m}$, i.e., smaller than the typical ML diameter. The $\mathrm{WSe}_{2} \mathrm{ML}$ is excited by a linearly polarized $\left(\sigma_{X}\right)$ continuous wave He-Ne laser $(1.96 \mathrm{eV})$ to generate valley coherence (i.e., optical alignment of excitons [45]). Our target is to detect the neutral exciton $X^{0}$ valley coherence in the linear basis in PL emission, see Fig. 1(b). A liquid crystal based linear polarization rotator is applied in the detection path, to detect a possible rotation of the linear basis of the PL signal with respect to the initial linear excitation basis. This approach avoids any macroscopic mechanical movement during the measurement and gives an accurate map of the angle dependent PL intensity as schematically illustrated in Fig. 1(a), the full data set is plotted in Fig. 1(d). The PL signal is dispersed in a spectrometer and detected with a Si-CCD camera. Based on these time integrated PL results, we can, then, generate the polar plot of the intensity of the $X^{0}$ emission for different magnetic field values and, then, monitor the rotation of the valley coherence by an angle $\theta$ as illustrated in Fig. 1(a). Faraday effects of the optical setup in applied fields have been systematically calibrated for plotting the valley coherence rotation angle.

Results and discussion.-Our experiment consists of three steps: First, we want to optically initialize a coherent superposition of exciton states. Second, during the exciton lifetime, we want to rotate the exciton pseudospin. Third,
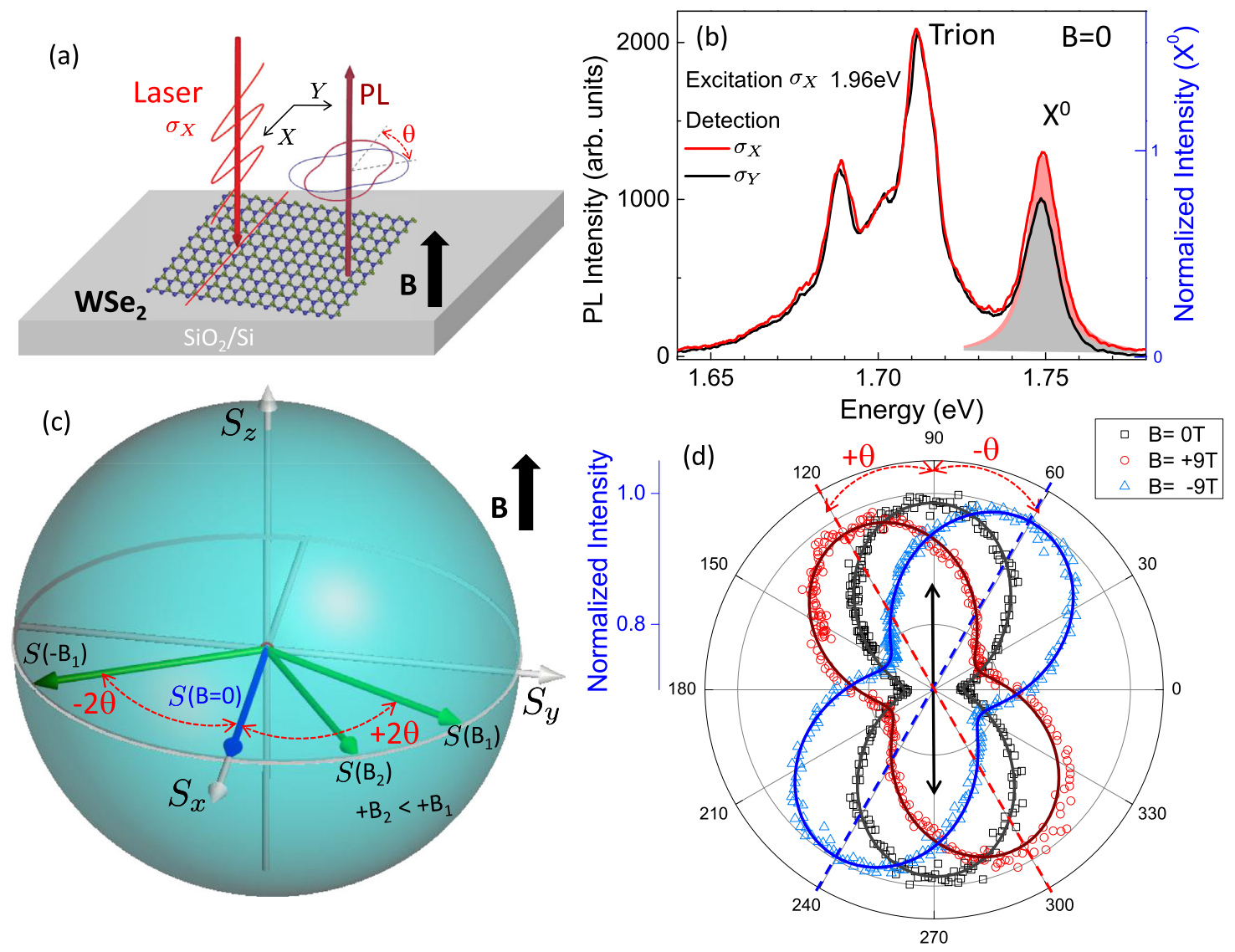

FIG. 1. (a) Schematic of experimental configuration. (b) Valley coherence generation following linearly polarized excitation $\sigma_{X}$ at $1.96 \mathrm{eV}$ probed by through PL emission of $X^{0}$ at the typical energy $1.75 \mathrm{eV}$ [31] at $T=4 \mathrm{~K}$. The trion emission and the low energy emission probably linked to localized states are, as expected, unpolarized [30]. (c) Representation of valley coherence (exciton pseudospin) on the Bloch sphere. At $B=0$, no rotation around the equator occurs as shown by the blue arrows. For $B \neq 0$, the exciton pseudospin precesses around the equator during the exciton lifetime. As PL emission times are very short (of the order of $\tau \approx 1$ ps), we probe the pseudospin orientation within the first precession period. The green arrows correspond to the state at a time $\tau$ after initialization, when recombination (pseudospin readout) occurs. By changing the amplitude and the direction of the magnetic field in the $Z$ direction, the valley coherence can be tuned to different points on the equator, compare $S\left(B_{1}\right)$ with $S\left(B_{2}\right)$. (d) The normalized $X^{0}$ angle dependent intensity polar plots for $B=0$ (black), $B=+9 \mathrm{~T}$ (red), and $B=-9 \mathrm{~T}$ (blue). The normalized intensity 0.7 corresponds to the center and 1 to the outermost gray circle. The laser polarization direction (constant for all $B$-field values) is indicated by the black arrow. 
we read out the final state after rotation. This three step experiment will, of course, only be successful if the coherence time is sufficiently long compared to the readout time [17]. In the experiment, we monitor the neutral exciton $X^{0}$ PL emission, which is linearly polarized along the same axis as the excitation laser. This corresponds to successful valley coherence generation [30], as shown in Fig. 1(b). The linear polarization of $X^{0}$ measured along the initial excitation direction $X$ (i.e., the laser polarization axis) is around $15 \%$. In contrast, both trion and lower energy emission peaks present no linear polarization as reported commonly for $\mathrm{ML} \mathrm{WSe} \mathrm{W}_{2}$ [30,31]. In analogy to spin, the average of this coherent superposition of valley states can be represented by a vector that lies on the equator of a Bloch sphere as illustrated in Fig. 1(c). The north $+S_{z}$ (south $-S_{z}$ ) pole on the Bloch sphere corresponds to a $|+1\rangle(|-1\rangle)$ exciton state, which can be optically generated by $\sigma^{+}\left(\sigma^{-}\right)$optical excitation. The equator corresponds to an $X^{0}$ in-plane valley pseudospin, coherent superposition of $|+1\rangle$ and $|-1\rangle$ with different phases. Radiative recombination of the $X^{0}$ results in photon emission. Importantly, the exciton's pseudospin direction on the equator will determine the linear polarization of the photon. In the absence of external fields, the linear polarization basis of the emitted photon is the same as the excitation laser; i.e., no pseudospin rotation occurs during the short PL emission time $\tau \approx 1$ ps [46-49] as the main axis of the polar plot Fig. 1(d) (black squares) is aligned along the laser polarization direction.

Our target is to go beyond a characterization of the created valley state and to create a new state by rotating the exciton spin. To this aim, we now apply a magnetic field $B$ which lifts valley degeneracy. Clear rotations of the linear polarization axis are observed in Fig. 1(d) for $B=-9 \mathrm{~T}$ and, also, $B=+9 \mathrm{~T}$. At $B=+9 \mathrm{~T}$ we measure a rotation of $\theta=$ $27^{\circ} \pm 4^{\circ}$ with respect to the initial laser excitation polarization. Changing from +9 to $-9 \mathrm{~T}$, we observe that $\theta$ also changes sign while keeping the same amplitude, as the exciton pseudospin rotation now occurs in the opposite direction. These trends are confirmed for the intermediate values at $B=$ $\pm 4 \mathrm{~T}$ as plotted in Fig. 2(a). Very similar data have been obtained for other ML $\mathrm{WSe}_{2}$ samples. The solid lines in Fig. 1(d) are fits using $r=A_{0}+A_{1} \cos [2(x-\theta)]$, where $x$ is the detection angle and $\theta$ represents the $X^{0}$ linear polarization angle. Experimentally, it is clear from Figs. 1(d) and 2(a) that we can control the rotation of the linearly polarized exciton emission with respect to the initial laser excitation axis.

Our next target is to show that the measured rotation angles are in agreement with a very simple interpretation of the experiments in terms of rotating a superposition of exciton $|+1\rangle$ and $|-1\rangle$ states. Our numerical estimations are based on exciton $g$ factors, exciton PL emission times, and valley coherence times from the literature and calibration experiments. At $B \neq 0$, the exciton valley coherence state, after a linearly polarized excitation along the $X$ direction, evolves with time as

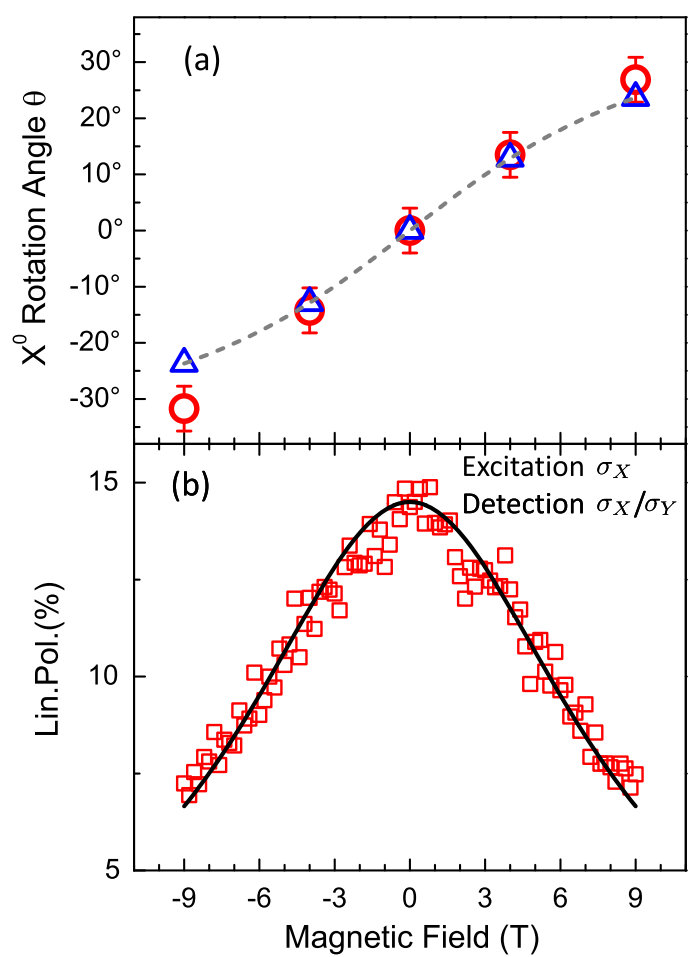

FIG. 2. (a) Absolute rotation angle of linear polarization of $X^{0}$ PL for different magnetic field values (red open circles). The gray dashed curve is calculated using $\theta=\left[\arctan \left(\Omega / T_{S 2}^{*}\right) / 2\right]$ and they are stressed by the blue open triangles at the experimental magnetic field for comparison. (b) The linear polarization of $X_{0}$ measured along the fixed direction $X$ and $Y$. The black solid line is the calculated value. Both the gray dashed line and the blue opened triangle in (a) and the black solid line in (b) are calculated by $g=-3.7, T_{2}^{*}=0.37 \mathrm{ps}$.

$1 / \sqrt{2}\left(|+1\rangle e^{-i \Omega t / 2}+|-1\rangle e^{i \Omega t / 2}\right)$, where $| \pm 1\rangle$ are the exciton spin states, the energy difference between the two $K$ valleys $\hbar \Omega=g \mu_{B} B$ with $\mu_{B}$ the Bohr magneton and $g$ the $X^{0}$ Landé $g$ factor. The new state vector on the equator of the Bloch sphere is shown in Fig. 1(c) by the green arrows labeled with $\pm B_{1}$. With a different magnetic field $B_{2}$, the final position of the valley coherence state can be selectively adjusted to a different rotation angle. From the measurements in Fig. 1(d), we determine the magnetic field dependence of the rotation angle, shown in Fig. 2(a) with open red circles. In our experiment, the initial pseudospin generated along the $X$ direction (laser polarization axis) precesses at frequency $\Omega$ around the magnetic field $B$ applied in the $Z$ direction (perpendicular to the ML plane). This scenario is very similar to the standard Hanle effect [50], which allows us to apply the same formalism [33-35]. For linear excitation, the generated pseudospin component $S_{z}=0$. In stationary conditions, the pseudospin state in-plane components in an applied magnetic field $B$ can be expressed as

$$
S_{x}(B)=\frac{S_{x}(0)}{1+\left(\Omega T_{S 2}^{*}\right)^{2}},
$$




$$
S_{y}(B)=\frac{\Omega T_{S 2}^{*}}{1+\left(\Omega T_{S 2}^{*}\right)^{2}} S_{x}(0)
$$

where, $1 / T_{S 2}^{*}=1 / \tau+1 / T_{S 2}, \tau$ is the exciton lifetime, $T_{S 2}$ is the exciton valley coherence time. The pseudospin rotates by an angle $\phi$, where $\tan \phi=\Omega T_{S 2}^{*}$. Now, we can estimate the $X^{0}$ PL rotation angle $\theta=\phi / 2$ and compare with our measured values summarized in Fig. 2(a). To calculate $\theta$, we use an exciton $g$ factor of $g=-3.7$ that we measured for $\mathrm{WSe}_{2} \mathrm{MLs}$ [41], which is similar to other reports for this material [37,51]. This leaves $T_{S 2}^{*}$ as an adjustable parameter, and we find excellent agreement with our data for $T_{S 2}^{*}=0.37 \mathrm{ps}$. The values for $\theta$ calculated at the magnetic field values used in our experiment are plotted as blue open triangles for comparison with the data, a general trend is shown as the gray dashed line. Assuming an exciton lifetime of $\tau \approx 2 \mathrm{ps}$ [47], in our nonresonant experiments, we can roughly estimate a valley coherence time $T_{S 2}$ of around $0.45 \mathrm{ps}$. This value is of the same order of magnitude as the measured valley coherence time of a highly crystalline CVD-grown $\mathrm{WSe}_{2}$ sample on a sapphire substrate [17]. This simple model yields excellent agreement with our experimental results, using a realistic value for $T_{S 2}^{*}$. Several physical mechanisms will limit the exciton coherence time $T_{S 2}$. The long-range Coulomb exchange interaction couples $|+1\rangle$ and $|-1\rangle$ exciton states and will, therefore, contribute to valley decoherence $[17,52-55]$. Also, the presence of additional carriers in the sample will induce scattering events that limit coherence times, here, experiments on excitons in the presence or absence of excess carriers in charge tunable structures will shed light on the stability of valley coherence [56].

We have shown that the linear polarization axis of the $X^{0}$ PL rotates as a function of the applied magnetic field. Using the linear polarization basis of the laser also for detection of the PL at $B \neq 0$, would lead, therefore, to a lowering of the observed linear polarization. This is exactly what has been reported recently for ML WSe 2 [39,41]. For the same sample investigated first in Figs. 1(d) and 2(a) (where we rotate the detection basis), we show, in Fig. 2(b), the linear polarization degree of the $X^{0} \mathrm{PL}$ for a fixed linear basis (parallel to the excitation laser). The linear polarization degree of $X^{0}$ drops from $15 \%$ at $B=0$ to $7 \%$ at $B \pm 9 \mathrm{~T}$. In our very simple description, without considering the magnetic field dependence of the valley coherence generation rate, this linear polarization should be directly deduced by Eq. (1). In Fig. 2(b), (fixed detection basis), we generate the solid line with the same parameters as used in Fig. 2(a) (optimized detection basis), the agreement of the simple model with the experimental results is remarkable. This close fit indicates that changes of the valley coherence generation rate as a function of magnetic field are negligible in our measurement.

Conclusions and perspectives.-Following the demonstration of optically generated valley polarization and valley coherence in the literature, we go a step further by demonstrating the coherent manipulation of valley states. This corresponds to a rotation of the exciton spin around the equator of the Bloch sphere, where the rotation angle is set by the value of the applied magnetic field. This is an important step towards the generation of an arbitrary exciton state in order to reach complete control of exciton states. To access states on the Bloch sphere away from the equator (i.e., $S_{z} \neq 0$ ), elliptically polarized light can be used for pseudospin initialization. In this case, the influence of the long-range electron-hole Coulomb exchange interaction on the exciton pseudospin evolution in applied fields needs to be investigated in the future [52-55]. The clearest signatures of valley coherence in $M X_{2}$ compounds have been observed in $\mathrm{ML} \mathrm{WSe}_{2}$, even using nonresonant excitation $[17,30,32]$. Another high quality material with spectrally narrow exciton emission is $\mathrm{ML} \mathrm{MoSe}_{2}$, but here, very close to resonant excitation is necessary to observe any valley polarization [57,58]. Only very recently, optically generated valley coherence has been observed in PL of acid treated $\mathrm{ML} \mathrm{MoS}_{2}$ [59], providing another interesting sample system for valley coherence experiments.

We acknowledge European Research Council Grant No. 306719 and Agence National de la Recherche Project MoS2ValleyControl for financial support. X. M. also acknowledges the Institut Universitaire de France. F. C and P. R acknowledge support from NEXT Grant No. ANR-10LABX-0037 in the framework of the Programme des Investissements d'Avenir. B. L. acknowledges the support by the National Science Foundation of China Grant No. 11574357 and the National Basic Research Program of China Grant No. 2015CB921001, the National Key Research Program of China Grant No. 2016YFA0300601. We acknowledge very fruitful discussions with Professor Junren Shi at early stages of the project.

Note added in proof.-For very recent work on valley states using related techniques see Refs. [60,61].

*blliu@iphy.ac.cn

†urbaszek@insa-toulouse.fr

[1] A. K. Geim and I. V. Grigorieva, Nature (London) 499, 419 (2013).

[2] K. S. Novoselov, D. Jiang, F. Schedin, T. J. Booth, V. V. Khotkevich, S. V. Morozov, and A. K. Geim, Proc. Natl. Acad. Sci. U.S.A. 102, 10451 (2005).

[3] A. Castellanos-Gomez, Nat. Photonics 10, 202 (2016).

[4] K. F. Mak, C. Lee, J. Hone, J. Shan, and T. F. Heinz, Phys. Rev. Lett. 105, 136805 (2010).

[5] A. Splendiani, L. Sun, Y. Zhang, T. Li, J. Kim, C.-Y. Chim, G. Galli, and F. Wang, Nano Lett. 10, 1271 (2010).

[6] G. Eda, H. Yamaguchi, D. Voiry, T. Fujita, M. Chen, and M. Chhowalla, Nano Lett. 11, 5111 (2011).

[7] W. Zhao, Z. Ghorannevis, L. Chu, M. Toh, C. Kloc, P.-H. Tan, and G. Eda, ACS Nano 7, 791 (2013).

[8] M. Amani, D.-H. Lien, D. Kiriya, J. Xiao, A. Azcatl, J. Noh, S. R. Madhvapathy, R. Addou, K. Santosh, M. Dubey et al., Science 350, 1065 (2015). 
[9] R. S. Sundaram, M. Engel, A. Lombardo, R. Krupke, A. C. Ferrari, P. Avouris, and M. Steiner, Nano Lett. 13, 1416 (2013).

[10] A. Pospischil, M. M. Furchi, and T. Mueller, Nat. Nanotechnol. 9, 257 (2014).

[11] F. Withers, O. D. Pozo-Zamudio, S. Schwarz, S. Dufferwiel, P. M. Walker, T. Godde, A. P. Rooney, A. Gholinia, C. R. Woods, P. Blake et al., Nano Lett. 15, 8223 (2015).

[12] H. J. Conley, B. Wang, J. I. Ziegler, R. F. Haglund, Jr., S. T. Pantelides, and K. I. Bolotin, Nano Lett. 13, 3626 (2013).

[13] D. Xiao, G.-B. Liu, W. Feng, X. Xu, and W. Yao, Phys. Rev. Lett. 108, 196802 (2012).

[14] X. Xu, D. Xiao, T. F. Heinz, and W. Yao, Nat. Phys. 10, 343 (2014).

[15] K. F. Mak, K. L. McGill, J. Park, and P. L. McEuen, Science 344, 1489 (2014).

[16] L. Yang, N. A. Sinitsyn, W. Chen, J. Yuan, J. Zhang, J. Lou, and S. A. Crooker, Nat. Phys. 11, 830 (2015).

[17] K. Hao, G. Moody, F. Wu, C. K. Dass, L. Xu, C.-H. Chen, M.-Y. Li, L.-J. Li, A. H. MacDonald, and X. Li, Nat. Phys. 12, 677 (2016).

[18] K. F. Mak and J. Shan, Nat. Photonics 10, 216 (2016).

[19] A. Chernikov, T. C. Berkelbach, H. M. Hill, A. Rigosi, Y. Li, O. B. Aslan, D. R. Reichman, M. S. Hybertsen, and T. F. Heinz, Phys. Rev. Lett. 113, 076802 (2014).

[20] B. Zhu, X. Chen, and X. Cui, Sci. Rep. 5, 9218 (2015).

[21] M. M. Ugeda, A. J. Bradley, S.-F. Shi, F. H. da Jornada, Y. Zhang, D. Y. Qiu, S.-K. Mo, Z. Hussain, Z.-X. Shen, F. Wang et al., Nat. Mater. 13, 1091 (2014).

[22] G. Wang, X. Marie, I. Gerber, T. Amand, D. Lagarde, L. Bouet, M. Vidal, A. Balocchi, and B. Urbaszek, Phys. Rev. Lett. 114, 097403 (2015).

[23] K. He, N. Kumar, L. Zhao, Z. Wang, K. F. Mak, H. Zhao, and J. Shan, Phys. Rev. Lett. 113, 026803 (2014).

[24] A. Hanbicki, M. Currie, G. Kioseoglou, A. Friedman, and B. Jonker, Solid State Commun. 203, 16 (2015).

[25] Z. Ye, T. Cao, K. O’Brien, H. Zhu, X. Yin, Y. Wang, S. G. Louie, and X. Zhang, Nature (London) 513, 214 (2014).

[26] K.F. Mak, K. He, J. Shan, and T. F. Heinz, Nat. Nanotechnol. 7, 494 (2012).

[27] H. Zeng, J. Dai, W. Yao, D. Xiao, and X. Cui, Nat. Nanotechnol. 7, 490 (2012).

[28] T. Cao, G. Wang, W. Han, H. Ye, C. Zhu, J. Shi, Q. Niu, P. Tan, E. Wang, B. Liu et al., Nat. Commun. 3, 887 (2012).

[29] D. Press, T. D. Ladd, B. Zhang, and Y. Yamamoto, Nature (London) 456, 218 (2008).

[30] A. M. Jones, H. Yu, N. J. Ghimire, S. Wu, G. Aivazian, J. S. Ross, B. Zhao, J. Yan, D. G. Mandrus, D. Xiao et al., Nat. Nanotechnol. 8, 634 (2013).

[31] G. Wang, L. Bouet, D. Lagarde, M. Vidal, A. Balocchi, T. Amand, X. Marie, and B. Urbaszek, Phys. Rev. B 90, 075413 (2014).

[32] G. Wang, M. M. Glazov, C. Robert, T. Amand, X. Marie, and B. Urbaszek, Phys. Rev. Lett. 115, 117401 (2015).

[33] S. Bar-Ad and I. Bar-Joseph, Phys. Rev. Lett. 66, 2491 (1991).

[34] T. Amand, X. Marie, P. Le Jeune, M. Brousseau, D. Robart, J. Barrau, and R. Planel, Phys. Rev. Lett. 78, 1355 (1997).

[35] X. Marie, P. Le Jeune, T. Amand, M. Brousseau, J. Barrau, M. Paillard, and R. Planel, Phys. Rev. Lett. 79, 3222 (1997).

[36] Y. Li, J. Ludwig, T. Low, A. Chernikov, X. Cui, G. Arefe, Y. D. Kim, A. M. van der Zande, A. Rigosi, H. M. Hill et al., Phys. Rev. Lett. 113, 266804 (2014).
[37] A. Srivastava, M. Sidler, A. V. Allain, D. S. Lembke, A. Kis, and A. Imamoğlu, Nat. Phys. 11, 141 (2015).

[38] D. MacNeill, C. Heikes, K. F. Mak, Z. Anderson, A. Kormányos, V. Zólyomi, J. Park, and D. C. Ralph, Phys. Rev. Lett. 114, 037401 (2015).

[39] G. Aivazian, Z. Gong, A. M. Jones, R.-L. Chu, J. Yan, D. G. Mandrus, C. Zhang, D. Cobden, W. Yao, and X. Xu, Nat. Phys. 11, 148 (2015).

[40] A. Arora, R. Schmidt, R. Schneider, M. R. Molas, I. Breslavetz, M. Potemski, and R. Bratschitsch, Nano Lett. 16, 3624 (2016).

[41] G. Wang, L. Bouet, M. M. Glazov, T. Amand, E. L. Ivchenko, E. Palleau, X. Marie, and B. Urbaszek, 2D Mater. 2, 034002 (2015).

[42] J. Kim, X. Hong, C. Jin, S.-F. Shi, C.-Y. S. Chang, M.-H. Chiu, L.-J. Li, and F. Wang, Science 346, 1205 (2014).

[43] E. J. Sie, J. W. McIver, Y.-H. Lee, L. Fu, J. Kong, and N. Gedik, Nat. Mater. 14, 290 (2015).

[44] R. L. Shoemaker and F. A. Hopf, Phys. Rev. Lett. 33, 1527 (1974).

[45] F. Meier and B. Zakharchenya, Modern Problems in Condensed Matter Sciences, Vol. 8 (North-Holland, Amsterdam 1984).

[46] M. Palummo, M. Bernardi, and J. C. Grossman, Nano Lett. 15, 2794 (2015).

[47] C. Robert, D. Lagarde, F. Cadiz, G. Wang, B. Lassagne, T. Amand, A. Balocchi, P. Renucci, S. Tongay, B. Urbaszek et al., Phys. Rev. B 93, 205423 (2016).

[48] C. Pöllmann, P. Steinleitner, U. Leierseder, P. Nagler, G. Plechinger, M. Porer, R. Bratschitsch, C. Schüller, T. Korn, and R. Huber, Nat. Mater. 14, 889 (2015).

[49] T. Korn, S. Heydrich, M. Hirmer, J. Schmutzler, and C. Schüller, Appl. Phys. Lett. 99, 102109 (2011).

[50] M. Dyakonov, in Spin Physics in Semiconductors, Springer Series in Solid-State Science, Vol. 157 (Springer-Verlag Berlin, 2008).

[51] A. Mitioglu, P. Plochocka, A. Granados del Aguila, P. Christianen, G. Deligeorgis, S. Anghel, L. Kulyuk, and D. Maude, Nano Lett. 15, 4387 (2015).

[52] M. Z. Maialle, E. A. de Andrada e Silva, and L. J. Sham, Phys. Rev. B 47, 15776 (1993).

[53] H. Yu, G. Liu, P. Gong, X. Xu, and W. Yao, Nat. Commun. 5, 3876 (2014).

[54] T. Yu and M. W. Wu, Phys. Rev. B 89, 205303 (2014).

[55] M. M. Glazov, T. Amand, X. Marie, D. Lagarde, L. Bouet, and B. Urbaszek, Phys. Rev. B 89, 201302 (2014).

[56] A. Chernikov, A. M. van der Zande, H. M. Hill, A. F. Rigosi, A. Velauthapillai, J. Hone, and T. F. Heinz, Phys. Rev. Lett. 115, 126802 (2015).

[57] G. Kioseoglou, A. T. Hanbicki, M. Currie, A. L. Friedman, and B. T. Jonker, Sci. Rep. 6, 25041 (2016).

[58] G. Wang, E. Palleau, T. Amand, S. Tongay, X. Marie, and B. Urbaszek, Appl. Phys. Lett. 106, 112101 (2015).

[59] F. Cadiz, S. Tricard, M. Gay, D. Lagarde, G. Wang, C. Robert, P. Renucci, B. Urbaszek, and X. Marie, Appl. Phys. Lett. 108, 251106 (2016).

[60] Z. Ye, D. Sun, T. F. Heinz, arXiv:1606.06806.

[61] R. Schmidt et al., Phys. Rev. Lett. 117, 077402 (2016). 